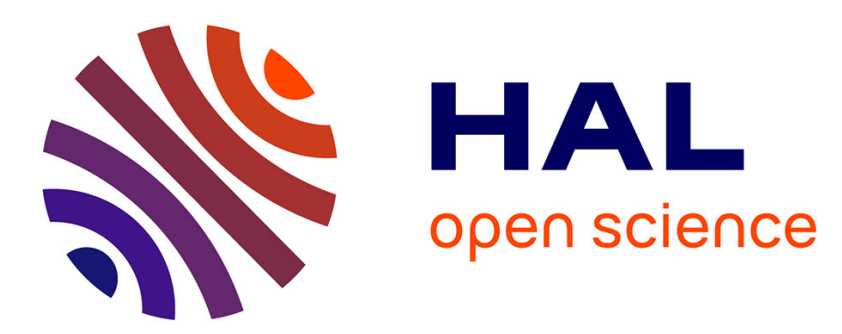

\title{
Models of stochastic gene expression and Weyl algebra
}

Samuel Alexandre Vidal, Michel Petitot, François Boulier, François Lemaire, Celine Kuttler

\section{To cite this version:}

Samuel Alexandre Vidal, Michel Petitot, François Boulier, François Lemaire, Celine Kuttler. Models of stochastic gene expression and Weyl algebra. Algebraic and Numeric Biology (ANB 2010), Jul 2010, Hagenberg, Austria. pp.76-97. hal-00492438v2

\section{HAL Id: hal-00492438 \\ https://hal.science/hal-00492438v2}

Submitted on 25 Jun 2011

HAL is a multi-disciplinary open access archive for the deposit and dissemination of scientific research documents, whether they are published or not. The documents may come from teaching and research institutions in France or abroad, or from public or private research centers.
L'archive ouverte pluridisciplinaire HAL, est destinée au dépôt et à la diffusion de documents scientifiques de niveau recherche, publiés ou non, émanant des établissements d'enseignement et de recherche français ou étrangers, des laboratoires publics ou privés. 


\title{
Models of Stochastic Gene Expression and Weyl Algebra
}

\author{
Samuel Vidal ${ }^{1}$, Michel Petitot ${ }^{2}$, François Boulier ${ }^{2}$, \\ François Lemaire $^{2}$, and Céline Kuttler ${ }^{2,3}$ \\ 1 Univ. Lille I \\ Lab. Paul Painlevé \\ samuel.vidal@math.univ-lille1.fr \\ ${ }^{2}$ Univ. Lille I \\ LIFL \\ \{michel.petitot,francois.boulier, \\ francois.lemaire, celine.kuttler\}@lifl.fr \\ 3 Univ. Lille I \\ IRI
}

\begin{abstract}
This paper presents a symbolic algorithm for computing the ODE systems which describe the evolution of the moments associated to a chemical reaction system, considered from a stochastic point of view. The algorithm, which is formulated in the Weyl algebra, seems more efficient than the corresponding method, based on partial derivatives. In particular, an efficient method for handling conservation laws is presented. The output of the algorithm can be used for a further investigation of the system behaviour, by numerical methods. Relevant examples are carried out.
\end{abstract}

Keywords: Stochastic models, Weyl algebra, Generating series.

\section{Introduction}

This paper is concerned with the modeling of gene regulatory networks by chemical reaction systems, from a stochastic point of view. To such systems, it is possible to associate a time varying random variable which counts the numbers of molecules of the various chemical species. It is well-known that the evolution, over time $t$, of the moments (mean, variance, covariance) of this random variable, may be described by a system of ordinary differential equations (ODEs), at least for first order chemical reaction systems $[6,16]$. See also [13] for an introduction to these topics. These systems of ODEs can be built from the probability generating function associated to a given chemical reaction system, by performing, essentially, the three following steps:

1. compute a Schrödinger equation analog [4, Eq. (5.60)] for the probability generating function $\phi(t, z)$, where $t$ denotes the time and $z$ denotes a vector of formal variables ; 
2. compute iterated derivatives of this equation, with respect to $t$;

3. evaluate the differentiated equation at $z=1$.

This paper shows how to build these ODE systems by using Weyl algebra methods. The idea consists in formulating the Schrödinger equation analog using Euler derivation operators (of the form $z \partial / \partial z$ ) instead of more traditional partial derivatives (of the form $\partial / \partial z$ ). As far as we know, the use of Weyl algebra in this context is new. It leads to a new algorithm which seems more efficient than the equivalent method, based on the use of partial derivatives. This last claim is not proved in this paper. It was suggested to us by the following observations. The formulation in the Weyl algebra permits to:

1. "combine in one step" steps 2 and 3 above, and thereby reduce the expression swell produced by step 2 (Formula (14) in Proposition 2) ;

2. prove the Formula (14), which we find more compact and simpler than the formulas in $[6,16]$.

3. allows to encode, in the Schrödinger equation analog, the linear conservation laws of the system (Algorithm of Section 5.1), and thereby take advantage of them at the very first step of the method;

A software prototype has been developed by the second author in the MAPLE computer algebra software. Supplementary related data are available at the url http://www.lifl.fr/ petitot/recherche/exposes/ANB2010.

The paper is organized as follows. In Section 2, the classical theory is recalled. The material can essentially be found (often piecewise) in many texts such as [4, chapter 5] or [8]. We feel the need to recall it in order to avoid confusions, since, depending on slight variants of the underlying assumptions, or slight variants of notations, different formula may be obtained. Our presentation is based on stochastic Petri nets [14,17]. In Section 3, the Weyl algebra and Euler operators are introduced, and the Schrödinger equation analog is reformulated, in this setting. In Section 4, the construction of the ODE system for the moments, from the differential operators, is explained (Proposition 1) and the algorithm is stated. In Section 5, the method for simplifying the Schrödinger equation analog using the conservation laws is provided. Section 6 provides a, new, combined formula for steps 1 and 2 (Formula (14) in Proposition 2). Some properties of the algorithm, which depend on the order of the chemical reaction system under study are explored in Section 7. In particular, some well-known results (related to compartmental models) are recovered from the Weyl algebra theory. Some examples are carried out in Section 8. The problem of the "infinite cascade" is studied for second order systems. Many parts of this article can be found in [15, Annexe A].

\section{The Classical Theory}

\subsection{Chemical Reactions Systems}

Definition 1. A chemical reaction system is given by a set of chemical species $\left(R_{1}, R_{2}, \ldots, R_{n}\right)$, and, a set of chemical reactions, of the following form, where 
$\alpha=\left(\alpha_{1}, \alpha_{2}, \ldots, \alpha_{n}\right), \beta=\left(\beta_{1}, \beta_{2}, \ldots, \beta_{n}\right)$ are multi-indices of nonnegative integers and $c$ is a positive real valued kinetic constant ${ }^{4}$.

$$
\alpha_{1} R_{1}+\cdots+\alpha_{n} R_{n} \stackrel{c}{\longrightarrow} \beta_{1} R_{1}+\cdots+\beta_{n} R_{n}
$$

Such a system, denoted $\mathcal{R}$, involves several reactions in general ; therefore it is a finite set of triples $(c, \alpha, \beta) \in \mathbb{R}_{>0} \times \mathbb{N}^{n} \times \mathbb{N}^{n}$. The state of the system $\nu=\left(\nu_{1}, \nu_{2}, \ldots, \nu_{n}\right) \in \mathbb{N}^{n}$ is the number of molecules of the $n$ chemical species at a given time. When reaction (1) occurs, the state vector instantaneously changes from the current value $\nu$ to the new value $\nu^{\prime}=\nu-\alpha+\beta$. The vector $\alpha$ indicates the quantities of the various species being consumed by the reaction while the vector $\beta$ indicates the produced quantities. For a reaction to occur it is necessary that $\alpha \leq \nu$, i.e. $\alpha_{i} \leq \nu_{i}$ for $i=1 \ldots n$. An example is provided by the following system, which is encoded by the set $\mathcal{R}=\{(\lambda,(2,1,0,0),(0,0,1,0)),(\mu,(0,0,1,0),(0,0,0,3))\}$.

$$
2 R_{1}+R_{2} \stackrel{\lambda}{\longrightarrow} R_{3}, \quad R_{3} \stackrel{\mu}{\longrightarrow} 3 R_{4} .
$$

\subsection{Stochastic Petri Nets}

Definition 2. A Petri net is a finite directed bipartite graph. Its nodes represent transitions (i.e. events that may occur, represented by boxes) and places (i.e. conditions for the events, represented by circles).

Denote $\mathcal{P}=\left\{p_{1}, p_{2}, \ldots, p_{n}\right\}$ the set of places and $\mathcal{T}=\left\{t_{1}, t_{2}, \ldots, t_{m}\right\}$ the set of transitions. At every instant, the place $p_{k},(1 \leq k \leq n)$ is supposed to contain $\nu_{k}$ indistinguishable tokens. The state vector $\nu=\left(\nu_{1}, \nu_{2}, \ldots, \nu_{n}\right) \in \mathbb{N}^{n}$ is the number of tokens present in the places. One denotes $C^{-}$and $C^{+}$the integer matrices where $C_{i, j}^{-}$is the number of arrows going from place $p_{j}$ to transition $t_{i}$, and $C_{i, j}^{+}$is the number of arrows going from transition $t_{i}$ to place $p_{j}$. The incidence matrix is $C=C^{+}-C^{-}$.

Definition 3. A stochastic Petri net is a Petri net endowed with a function $\rho: \mathcal{T} \longrightarrow \mathbb{R}_{>0}$.

The category of the stochastic Petri nets is strictly equivalent to the category of chemical reaction systems. To each transition $t_{i} \in \mathcal{T}$, a chemical reaction like (1) is associated. It is coded by a triple $(c, \alpha, \beta) \in \mathbb{R}_{>0} \times \mathbb{N}^{n} \times \mathbb{N}^{n}$ with $\alpha=\left(C_{i j}^{-}\right)_{j=1 \ldots n}, \beta=\left(C_{i j}^{+}\right)_{j=1 \ldots n}$ and $c=\rho\left(t_{i}\right)$. The incidence matrix $C$ is then the transpose of the stoichiometry matrix of the chemical reaction system. The correspondence holds:

$$
\begin{aligned}
\text { token } & \longleftrightarrow \text { chemical molecule } \\
\text { place } & \longleftrightarrow \text { chemical species } \\
\text { transition } & \longleftrightarrow \text { chemical reaction }
\end{aligned}
$$

\footnotetext{
${ }^{4}$ Called stochastic reaction constant in [7].
} 
The chemical reaction system (2) corresponds to the Petri net of Figure 1 with the associated matrices

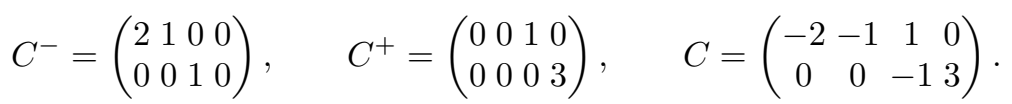

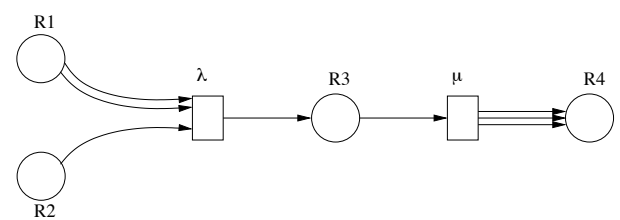

Fig. 1. Petri net of Example (2)

\subsection{Markov Chain of the Temporisation and Master Equation}

One gives the "standard temporisation" of a stochastic Petri net by associating it with a continuous time Markov chain $\left\{N(t) ; t \in \mathbb{R}_{\geq 0}\right\}$ where the vector valued random variable $N(t)$ counts, at time $t$, the number of tokens in the places of the network. Let $\pi_{\nu}(t)$ be the probability that the process is in state $N(t)=\nu$ at time $t$. The master-equation [5] of a Markov chain is a linear differential system governing the evolution, over time, of the row vector $\pi(t)=\left(\pi_{\nu}(t) ; \nu \in \mathbb{N}^{n}\right)$. It is written as follows:

$$
\frac{d}{d t} \pi(t)=\pi(t) A
$$

For each couple of multi-indices $(\alpha, \nu) \in \mathbb{N}^{n} \times \mathbb{N}^{n}$, the following product of binomial coefficients is defined

$$
\left(\begin{array}{c}
\nu \\
\alpha
\end{array}\right)=\left(\begin{array}{c}
\nu_{1} \\
\alpha_{1}
\end{array}\right)\left(\begin{array}{c}
\nu_{2} \\
\alpha_{2}
\end{array}\right) \cdots\left(\begin{array}{c}
\nu_{n} \\
\alpha_{n}
\end{array}\right) .
$$

The Markov chain $\left\{N(t) ; t \in \mathbb{R}_{\geq 0}\right\}$ associated to the stochastic Petri net $\mathcal{R}$, is defined on the discrete state space $\mathbb{N}^{n}$. For each triple $(c, \alpha, \beta) \in \mathcal{R}$ and for each state $\nu \geq \alpha$ an arrow going from $\nu$ to $\nu^{\prime}=\nu-\alpha+\beta$ is built. It is labeled by the transition rate $c\left(\begin{array}{c}\nu \\ \alpha\end{array}\right)$. Schematically, this can be written:

$$
(\nu+\alpha-\beta) \stackrel{c\left({ }^{\nu+\alpha-\beta}\right)}{\longrightarrow}(\nu) \stackrel{c\left(\begin{array}{l}
\nu \\
\alpha
\end{array}\right)}{\longrightarrow}(\nu-\alpha+\beta) .
$$

Assume that the system is in state $\nu \in \mathbb{N}^{n}$ at time $t$. The probability that the chemical reaction $(c, \alpha, \beta)$ occurs within the time range $\left[t, t+\varepsilon\left[\right.\right.$ is $\varepsilon c\left(\begin{array}{l}\nu \\ \alpha\end{array}\right)+\mathrm{o}(\varepsilon)$. Then the master-equation (3) takes the form

$$
\frac{d}{d t} \pi_{\nu}(t)=\sum_{(c, \alpha, \beta) \in \mathcal{R}} c\left(\begin{array}{c}
\nu+\alpha-\beta \\
\alpha
\end{array}\right) \pi_{\nu+\alpha-\beta}(t)-c\left(\begin{array}{l}
\nu \\
\alpha
\end{array}\right) \pi_{\nu}(t) .
$$


According to Definition (4), the first term of the sum is zero whenever $\beta>\nu$ and the second is zero whenever $\alpha>\nu$. This differential system involves an infinite number of unknowns $\pi_{\nu}(t)$ constrained by an infinite linear differential system (see Example (8)).

\subsection{The Schrödinger Equation Analog}

For investigating models analytically we introduce the probability generating function [4, sect. 5.3]

$$
\phi(t, z)=\sum_{\nu \geq 0} \pi_{\nu}(t) z^{\nu} .
$$

Note that $\phi(t, z)$ is also equal to $\mathrm{E} z^{N(t)}$ (i.e. the mean value of $z^{N(t)}$ ) since $\mathrm{E} z^{N(t)}=\sum_{\nu \geq 0} \operatorname{prob}\left(z^{\nu}=z^{N(t)}\right) z^{\nu}$. Given any chemical reaction system, it is possible to compute a general equation for $\phi$ [4, Eq. (5.60)]. This general equation is a Schrödinger equation analog. The differential operator $H$ is a Hamiltonian.

$$
\frac{\partial}{\partial t} \phi(z, t)=H \phi(z, t) \quad \text { where } \quad H=\sum_{(c, \alpha, \beta) \in \mathcal{R}} \frac{c}{\alpha !}\left(z^{\beta}-z^{\alpha}\right)\left(\frac{\partial}{\partial z}\right)^{\alpha} .
$$

The Hamiltonian of Example (2) is

$$
H=\frac{1}{2} \lambda\left(z_{3}-z_{1}^{2} z_{2}\right) \frac{\partial^{2}}{\partial z_{1}^{2}} \frac{\partial}{\partial z_{2}}+\mu\left(z_{4}^{3}-z_{3}\right) \frac{\partial}{\partial z_{3}} .
$$

By differentiating the probability generating function (6) and evaluating it at $z=1$, i.e, at $z_{1}=\cdots=z_{n}=1$, formulas which bind $\phi$, the means and the variances of the number of molecules of the chemical species, are obtained. Here are a few examples. A mere evaluation yields: $\left.\phi(t, z)\right|_{z=1}=1$. Differentiating (6) with respect to any $z_{i}$ and evaluating at $z=1$ provides the expected value of the number of molecules of species $R_{i}$, i.e. $\mathrm{E} N_{i}(t)$

$$
\left(\frac{\partial}{\partial z_{i}} \phi(z, t)\right)_{\left.\right|_{z=1}}=\sum_{\nu} \nu_{i} \pi_{\nu}(t)=\operatorname{E} N_{i}(t) .
$$

Differentiating (6) twice with respect to some fixed $z_{i}$ and evaluating at $z=1$ provides a formula featuring the expected value of the square of the number of molecules of $R_{i}$, denoted $\mathrm{E} N_{i}(t)^{2}$, together with $\mathrm{E} N_{i}(t)$ :

$$
\begin{aligned}
\left(\frac{\partial}{\partial z_{i}} \frac{\partial}{\partial z_{i}} \phi(z, t)\right)_{\left.\right|_{z=1}} & =\sum_{\nu} \nu_{i}\left(\nu_{i}-1\right) \pi_{\nu}(t) \\
& =\sum_{\nu} \nu_{i}^{2} \pi_{\nu}(t)-\nu_{i} \pi_{\nu}(t)=\operatorname{E} N_{i}(t)^{2}-\operatorname{E} N_{i}(t) .
\end{aligned}
$$

The variance of the number of molecules of $R_{i}$ satisfies the well-known formula: $\operatorname{Var} N_{i}(t)=\mathrm{E} N_{i}(t)^{2}-\left(\mathrm{E} N_{i}(t)\right)^{2}$. The above formula can then be restated using $\mathrm{E} N_{i}(t)$ and $\operatorname{Var} N_{i}(t)$ only. Then, from the arguments above and 
the Schrödinger equation analog (7), an ODE system for the means and the variances $\mathrm{E} N_{i}(t)$ and $\operatorname{Var} N_{i}(t)$ can be computed. The method is illustrated over the following example:

$$
\emptyset \stackrel{\lambda}{\longrightarrow} R \quad R \stackrel{\mu}{\longrightarrow} \emptyset .
$$

It corresponds to the creation and degradation of mRNA by a unregulated gene. It also corresponds to the $M / M / \infty$ client-server system [5]. The following set of triples $\mathcal{R}=\{(\lambda,(0),(1)),(\mu,(1),(0))\}$ provides a description of that system. The Markov chain is described by the transition rates $\lambda\left(\begin{array}{l}\nu \\ 0\end{array}\right)=\lambda$ and $\mu\left(\begin{array}{l}\nu \\ 1\end{array}\right)=\mu \nu$ for all $\nu \in \mathbb{N}$. Using the convention $\pi_{-1}(t)=0$, the master-equation (5) of that

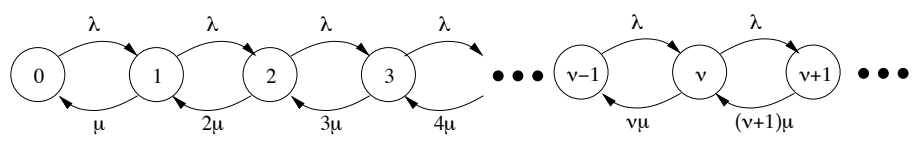

Fig. 2. The Markov chain associated to System (8)

Markov chain is:

$$
\frac{d}{d t} \pi_{\nu}(t)=\lambda\left[\pi_{\nu-1}(t)-\pi_{\nu}(t)\right]+\mu\left[(\nu+1) \pi_{\nu+1}(t)-\nu \pi_{\nu}(t)\right] \quad(\nu \geq 0) .
$$

The Schrödinger equation analog (7) is:

$\frac{\partial}{\partial t} \phi(t, z)=\left[\lambda(z-1)+\mu(1-z) \frac{\partial}{\partial z}\right] \phi(t, z)=\lambda(z-1) \phi(t, z)+\mu(1-z) \phi_{z}(t, z)$.

In order to compute an ODE for the mean $\mathrm{E} N(t)$, that relation is differentiated with respect to $z$,

$$
\frac{\partial}{\partial z} \frac{\partial}{\partial t} \phi(t, z)=\lambda\left[\phi(t, z)+(z-1) \phi_{z}(t, z)\right]+\mu\left[-\phi_{z}(t, z)+(1-z) \phi_{z z}(t, z)\right] .
$$

The partial derivatives $\frac{\partial}{\partial t}$ and $\frac{\partial}{\partial z}$ commute. At the point $z=1$, this equation becomes:

$$
\frac{\partial}{\partial t} \phi_{z}(t, z)_{\left.\right|_{z=1}}=\lambda-\mu \phi_{z}(t, z)_{\left.\right|_{z=1}} \quad \text { i.e. } \quad \frac{d}{d t} \mathrm{E} N(t)=\lambda-\mu \mathrm{E} N(t) .
$$

An ODE for $\mathrm{E} N(t)$ is obtained. Similar computations provide an ODE for $\operatorname{Var} N(t)$. The initial values are easily obtained since, at $t=0$, the expected value of $N(t)$ is equal to the initial quantity $n_{0}$ of the chemical species and the variance of $N(t)$ is zero. The analysis of that dynamics can be done numerically or symbolically, depending on the instance of the problem.

Using this method, an ODE for any moment $\operatorname{E} N^{q}(t)$, where $q \in \mathbb{N}$, can be computed. In the particular case of order 1 chemical reaction systems (Definition 6), the ODE system is finite and exact values of the means and the variances $[4$, sect. 5.3.3] can be computed. The above example has order 1. 
In the general case, the ODE system is infinite, since for any $q \in \mathbb{N}$, the evolutions of the moments of order $q$ depend on moments of higher order (problem of the infinite cascade $[6,16]$ ). It is sometimes possible to compute exact values for the means and the variances by ad hoc arguments. Otherwise, the ODE system needs to be truncated and provides a more or less usable approximation. The truncation, which is obtained by assuming that random variables $N_{i}(t)$ are independent, provides the classical deterministic model, which is used when the number of molecules is large.

\section{Reformulation in the Weyl Algebra}

In this section, the properties of the Weyl algebra [3] needed to understand the algorithm of Section 4 are introduced. Then, the construction of the Hamiltonian (7) is reformulated.

The Weyl algebra $\operatorname{Weyl}_{\mathbb{R}}\left(z_{1}, \ldots, z_{n}\right)=\mathbb{R}\left[z_{1}, z_{2}, \ldots, z_{n}\right]\left[\partial_{z_{1}}, \partial_{z_{2}}, \ldots, \partial_{z_{n}}\right]$ is the algebra of (polynomial) differential operators defined on the affine algebraic manifold $\mathbb{R}^{n}$. It is a non-commutative and associative algebra generated by the symbols $z_{k}$ and $\partial_{z_{k}}$ for $k=1 \ldots n$ constrained by the commutation relations $\left[\partial_{z_{i}}, z_{j}\right]=\partial_{z_{i}} z_{j}-z_{j} \partial_{z_{i}}=1$ if $i=j$, and 0 otherwise. The commutator (also called Lie Bracket) between $A$ and $B$, denoted $[A, B]$, is defined by the relation $[A, B]=A B-B A$. For all $\alpha, \beta \in \mathbb{N}^{n}$, denote $z^{\alpha}=z_{1}^{\alpha_{1}} z_{2}^{\alpha_{2}} \ldots z_{n}^{\alpha_{n}}$ and $\partial_{z}^{\beta}=\partial_{z_{1}}^{\beta_{1}} \partial_{z_{2}}^{\beta_{2}} \ldots \partial_{z_{n}}^{\beta_{n}}$. For each element $z^{\alpha} \partial_{z}^{\beta}$, define the degree $\operatorname{deg} z^{\alpha} \partial_{z}^{\beta}$ as $|\alpha|-|\beta|$, and the order ord $z^{\alpha} \partial_{z}^{\beta}$ as $|\beta|$, where $|\alpha|=\alpha_{1}+\alpha_{2}+\cdots+\alpha_{n}$ and $|\beta|=\beta_{1}+\beta_{2}+\cdots+\beta_{n}$.

We now define the evaluation of a differential operator. Any differential operator $D \in \operatorname{Weyl}_{\mathbb{R}}\left(z_{1}, \ldots, z_{n}\right)$ can be written in a unique way as a finite sum of terms $f_{\nu} \partial_{z}^{\nu}$ where $\nu \in \mathbb{N}^{n}$, and where $f_{\nu} \in \mathbb{R}\left[z_{1}, z_{2}, \ldots, z_{n}\right]$ defines a function $\mathbb{R}^{n} \rightarrow \mathbb{R}$. This allows to define the evaluation of $D$ at a point $p \in \mathbb{R}^{n}$ :

$$
D_{\left.\right|_{p}}=\sum_{\nu} f_{\nu}(p) \partial_{z}^{\nu}
$$

The operator $D_{\left.\right|_{p}}: \mathbb{R}\left[z_{1}, z_{2}, \ldots, z_{n}\right] \rightarrow \mathbb{R}$ is defined in a coordinate free way by setting $D_{\left.\right|_{p}}(h)=D(h)_{\left.\right|_{p}}$ for any function $h \in \mathbb{R}\left[z_{1}, z_{2}, \ldots, z_{n}\right]$. The term $D(h)_{\left.\right|_{p}}$ denotes the real number obtained by evaluating the function $D(h)$ at $p$. The evaluation does not commute with the multiplication of the algebra $\operatorname{Weyl}_{\mathbb{R}}(z)$.

We now reformulate the Schrödinger equation analog, using "falling powers" (also called falling factorials) and Euler operators. Falling powers are defined by $x \underline{p}=x(x-1)(x-2) \cdots(x-p+1)$, where $p \in \mathbb{N}$. Then, binomial coefficients can be reformulated as follows: $\left(\begin{array}{l}x \\ p\end{array}\right)=(1 / p !) x \underline{p}$.

Definition 4. Euler operators are defined by $\theta_{k}=z_{k} \partial / \partial z_{k}$.

For any $\alpha \in \mathbb{N}^{n}$, one defines $\theta \underline{\underline{\alpha}}=\theta_{1}^{\underline{\alpha}_{1}} \theta_{2}^{\underline{\alpha}_{2}} \cdots \theta_{n}^{\underline{\alpha}}$. The next lemma is classical:

Lemma 1. For any $\alpha \in \mathbb{N}^{n}$, one has $\theta^{\underline{\alpha}}=z^{\alpha}\left(\frac{\partial}{\partial z}\right)^{\alpha}$. 
Given any chemical reaction system, the Schrödinger equation analog (7) which governs the probability generating function (6) can be formulated in the Weyl algebra as follows. The formula can be justified using Formula (7) and Lemma 1. The Hamiltonian $H$ belongs to $\operatorname{Weyl}_{\mathbb{R}}(z)$. It is a linear operator acting on the formal series in the variables $\left(z_{1}, z_{2}, \ldots, z_{n}\right)$ :

$$
H=\sum_{(c, \alpha, \beta) \in \mathcal{R}} \frac{c}{\alpha !}\left(z^{\beta-\alpha}-1\right) \theta^{\underline{\alpha}} .
$$

The Hamiltonian of Example (2) can now be written as:

$$
H=\frac{1}{2} \lambda\left(\frac{z_{3}}{z_{1}^{2} z_{2}}-1\right) \theta_{1}\left(\theta_{1}-1\right) \theta_{2}+\mu\left(\frac{z_{4}^{3}}{z_{3}}-1\right) \theta_{3} .
$$

\section{The Algorithm}

This section presents our algorithm which computes the differential equations satisfied by the moments a stochastic Petri net (up to a certain order). First Proposition 1 is proved. Then our algorithm is stated and proved using Proposition 1.

Since $N(t)$ is a (time dependent) random variable taking values in $\mathbb{N}^{n}$, it is possible to consider any random variable of the form $f(N(t))$ where the function $f: \mathbb{N}^{n} \rightarrow \mathbb{R}$ is a polynomial function represented by an element $f \in \mathbb{R}\left[\theta_{1}, \theta_{2}, \ldots, \theta_{n}\right]$, i.e, a polynomial in the Euler operators. Observe that, by definition of $\theta_{k}$, the polynomial $f$ is also an element of the algebra $\operatorname{Weyl}_{\mathbb{R}}(z)$. For example, to the polynomial $f=\theta_{1}^{2} \theta_{2}+3 \theta_{1} \in \mathbb{R}\left[\theta_{1}, \theta_{2}\right]$, the following objects can be associated: the operator $f(\theta)=\theta_{1}^{2} \theta_{2}+3 \theta_{1} \in \operatorname{Weyl}_{\mathbb{R}}\left(z_{1}, z_{2}\right)$; the number $f(\nu)=\nu_{1}^{2} \nu_{2}+3 \nu_{1} \in \mathbb{R}$; the random variable $f(N(t))=N_{1}^{2}(t) N_{2}(t)+3 N_{1}(t)$ defined on $\mathbb{N}^{2}$ with value in $\mathbb{R}$; the commutator $[f(\theta), H]=f(\theta) H-H f(\theta)$; and the evaluated commutator, $[f(\theta), H]_{\left.\right|_{z_{1}=z_{2}=1}}$, which is a polynomial in $\mathbb{R}\left[\theta_{1}, \theta_{2}\right]$. The mean value of the random variable $f(N(t))$ is, by definition, equal to E $f(N(t))=\sum_{\nu} f(\nu) \pi_{\nu}(t)$.

Lemma 2. For any polynomial $f \in \mathbb{R}\left[\theta_{1}, \theta_{2}, \ldots, \theta_{n}\right]$, interpreted as a differential operator acting on the generating series $\phi(t, z)$, one has the relation: $\mathrm{E} f(N(t))=f(\theta) \quad \mathrm{E} z^{N(t)}{ }_{z=1}$.

Proof. For any element $\nu \in \mathbb{N}^{n}$, one has $f(\theta) z^{\nu}=f(\nu) z^{\nu}$. It follows that $f(\theta) \phi(t, z)=\sum_{\nu} f(\nu) \pi_{\nu}(t) z^{\nu}$. Thus, evaluating at the point $z=1$ leads to: $\left.f(\theta) \operatorname{E} z^{N(t)}\right|_{z=1}=\sum_{\nu} f(\nu) \pi_{\nu}(t)=\operatorname{E} f(N(t)$.

Proposition 1. Given $f \in \mathbb{R}\left[\theta_{1}, \theta_{2}, \ldots, \theta_{n}\right]$, denote $f_{H}(\theta)=[f(\theta), H]_{\left.\right|_{z=1}}$. Then

$$
\frac{d}{d t} \mathrm{E} f(N(t))=\mathrm{E} f_{H}(N(t)) .
$$


Proof. Start from the Schrödinger equation analog $\frac{\partial}{\partial t} \phi(t, z)=H \phi(t, z)$. The partial derivation $\partial / \partial t$ commutes with the operator $f(\theta)$ and the evaluation at $z=1$. Thus $\partial / \partial t f(\theta) \phi(t, z)_{\mid z=1}=f(\theta) H \phi(t, z)_{\mid z=1}$. The left-hand side of the equality is equal to $d / d t \mathrm{E} f(N(t))$ according to Lemma 2 . In the right-hand side, the product $f(\theta) H$ van be replaced by the commutator $[f(\theta), H]$ since, according to Formula (11), the Hamiltonian $H$ is zero at $z=1$. The second member is equal to $\mathrm{E} f_{H}(N(t))$ by Lemma 2 .

\section{The Algorithm}

Input: A stochastic Petri net $\mathcal{R}$ and a maximum order $q \in \mathbb{N}$.

Output: A linear differential system characterizing the time evolution of moments, up to degree $q$.

1. Compute the Hamiltonian $H$ of $\mathcal{R}$ using formula (11).

2. For all multi-indices $\kappa \in \mathbb{N}^{n}$ such that $|\kappa| \leq q$, compute the commutator evaluated at $z=1$

$$
f_{\kappa}=\left[\theta^{\kappa}, H\right]_{\left.\right|_{z=1}}, \quad\left(f_{\kappa} \in \mathbb{R}\left[\theta_{1}, \ldots, \theta_{n}\right]\right),
$$

Then generate the linear differential equation $\frac{d}{d t} \mathrm{E} N^{\kappa}(t)=\mathrm{E} f_{\kappa}(N(t))$, using Proposition 1.

The polynomial $f_{\kappa}$ in Formula (12) can be computed in different ways. It can be computed by using a smart computation of the Lie bracket (which avoids the expansion of the Lie bracket by computing $\theta^{\kappa} H$ and then substracting $H \theta^{\kappa}$ ) and a specialization to $z=1$. Otherwise, it is possible to compute $f_{\kappa}=\theta^{\kappa} H_{\mid z=1}$ since the term $H \theta^{\kappa}{ }_{z=1}$ cancels.

Remark 1. The algorithm is stated using Weyl algebras computations since it makes the proofs easier to establish. Section 6 also provides a formula only based on basic operations on commutative polynomials in $\theta$. As detailed in Section 6 , we believe that avoiding Weyl algebras computations is easier from a software implementation point of view.

The returned ODE system is truncated. Thus, some ODE may depend on moments of order higher than $q$, for which no ODE is generated. This problem does not occur in Example (8). The Hamiltonian is

$$
H=\lambda(z-1)+\mu\left(\frac{1}{z}-1\right) \theta \quad \text { with } \quad \theta=z \frac{\partial}{\partial z} .
$$

The algorithm computes the brackets

$$
[\theta, H]_{\left.\right|_{z=1}}=\lambda-\mu \theta, \quad\left[\theta^{2}, H\right]_{\left.\right|_{z=1}}=\lambda+(2 \lambda+\mu) \theta-2 \mu \theta^{2} .
$$

and returns the differential system

$$
\frac{d}{d t} \mathrm{E} N(t)=\lambda-\mu \mathrm{E} N(t), \quad \frac{d}{d t} \mathrm{E} N^{2}(t)=\lambda+(2 \lambda+\mu) \mathrm{E} N(t)-2 \mu \mathrm{E} N^{2}(t) .
$$


The variance is computed using $\operatorname{Var} N(t)=\mathrm{E} N^{2}(t)-(\mathrm{E} N(t))^{2}$. The dynamics of the mean $x(t)=\mathrm{E} N(t)$ and the variance $v(t)=\operatorname{Var} N(t)$ follows immediately.

$$
\frac{d}{d t} x(t)=\lambda-\mu x(t), \quad \frac{d}{d t} v(t)=\lambda+\mu x(t)-2 \mu v(t) .
$$

\section{Model Reduction, Model Restriction and Conservation Laws}

Definition 5. Let $\lambda$ be a $n$-dimensional vector of integers. A conservation law $I_{\lambda}$ is a linear combination of the following form, with integer coefficients, which is conserved by each transition of the considered Petri net:

$$
I_{\lambda}(\nu)=\lambda_{1} \nu_{1}+\lambda_{2} \nu_{2}+\cdots+\lambda_{n} \nu_{n}, \quad\left(\nu \in \mathbb{N}^{n}, \lambda \in \mathbb{Z}^{n}\right) .
$$

This notion is independent of the temporisation, hence of the kinetic constants associated to chemical reactions. Recall that the incidence matrix of a Petri net is the transpose of the stoichiometry matrix of a chemical reaction system. The next lemma is then well-known [9, sect. 5.3].

Lemma 3. Let $\mathcal{R}$ be a Petri net. The column vector $\lambda=\left(\lambda_{1}, \lambda_{2}, \ldots, \lambda_{n}\right) \in \mathbb{Z}^{n}$ defines a conservation law iff $C \lambda=0$ where $C=C^{+}-C^{-}$is the incidence matrix of $\mathcal{R}$.

\subsection{Model Reduction}

A conservation law $I_{\lambda}$ induces a graduation $\mathrm{w}_{\lambda}$ of the algebra Weyl $\mathrm{l}_{\mathbb{R}}(z)$ defined by $\mathrm{w}_{\lambda}\left(\partial / \partial z_{k}\right)=-\lambda_{k}$ and $\mathrm{w}_{\lambda}\left(z_{k}\right)=\lambda_{k}$ for all $k=1 \ldots n$. Define, moreover, $\mathrm{w}_{\lambda}\left(z^{\nu}\right)=\sum_{k} \lambda_{k} \nu_{k}$, that is $\mathrm{w}_{\lambda}\left(z^{\nu}\right)=I_{\lambda}(\nu)$, for all $\nu \in \mathbb{N}^{n}$.

Lemma 4. Assume that a conservation law $I_{\lambda}$ holds. Then, $\phi(t, z)$ is a formal power series, homogeneous for $\mathrm{w}_{\lambda}$, with weight $\mathrm{w}_{\lambda}(\phi(t, z))=I_{\lambda}\left(\nu_{0}\right)$, where the multi-index $\nu_{0} \in \mathbb{N}^{n}$ denotes the initial state at $t=0$.

Proof. As $I_{\lambda}(\nu)$ is independent of the time $t$, the fact that $I_{\lambda}(\nu) \neq I_{\lambda}\left(\nu_{0}\right)$ implies that $\pi_{\nu}(t)=0$ for any $t \geq 0$. As a consequence, the series $\phi(t, z)=\sum_{\nu} \pi_{\nu}(t) z^{\nu}$ is $\mathrm{w}_{\lambda}$-homogeneous of weight $\mathrm{w}_{\lambda}\left(z^{\nu_{0}}\right)$.

Denote $C_{0}=I_{\lambda}\left(\nu_{0}\right) \in \mathbb{Z}$. Then the operator $I_{\lambda}(\theta)-C_{0}=\sum_{k} \lambda_{k} \theta_{k}-C_{0}$ vanishes on the generating series $\phi(t, z)$ because, according to Lemma 4 , one has $I_{\lambda}(\theta) \phi(t, z)=C_{0} \phi(t, z)$. Every operator of the left ideal generated in $\operatorname{Weyl}_{\mathbb{R}}(z)$ by $I_{\lambda}(\theta)-C_{0}$ has therefore a null action on $\phi(t, z)$.

\section{The Model Reduction Algorithm}

Input: A Hamiltonian $H \in \operatorname{Weyl}_{\mathbb{R}}\left(z_{1}, \ldots, z_{n}\right)$ describing the evolution of the multi-index $N(t)=\left(N_{1}(t), \ldots, N_{n}(t)\right)$ and a conservation law $I_{\lambda}$. One assumes, without loss of generality, that $\lambda_{n}$ is nonzero. 
Output: A reduced Hamiltonian $H^{\prime} \in \operatorname{Weyl}_{\mathbb{R}}\left(z_{1}, \ldots, z_{n-1}\right)$ describing the evolution of $\left(N_{1}(t), \ldots, N_{n-1}(t)\right)$.

The new Hamiltonian $H^{\prime}$ is obtained from $H$ by the following substitution

$$
\theta_{n} \mapsto \frac{1}{\lambda_{n}}\left[C_{0}-\left(\lambda_{1} \theta_{1}+\lambda_{2} \theta_{2}+\cdots+\lambda_{n-1} \theta_{n-1}\right)\right], \quad z_{n} \mapsto 1
$$

\subsection{Model Restriction}

The presence of conservation laws often enables us to bound some random variables of the model. Those bounds in general depend on the initial state $\nu_{0} \in \mathbb{N}^{n}$. In this situation, it is possible to restrict the model by taking a quotient of the Weyl algebra $\mathrm{Weyl}_{\mathbb{R}}\left(z_{1}, \ldots, z_{n}\right)$ by a left ideal. The method is presented over the following system of chemical reactions

$$
R_{1}+R_{2} \stackrel{\lambda}{\longrightarrow} R_{3}, \quad R_{3} \stackrel{\mu}{\longrightarrow} R_{1}+R_{2},
$$

together with initial conditions $\nu^{0}=(a, b, 0)$ for $a, b \in \mathbb{N}$. The Petri net $\mathcal{R}$ admits two conservation laws $\nu_{1}+\nu_{3}=a$ and $\nu_{2}+\nu_{3}=b$. It is therefore possible to consider the random variable $N_{3}(t)$ only, with the bound $0 \leq N_{3}(t) \leq \min (a, b)$. Our software computes the Hamiltonian:

$$
H=\lambda\left(\frac{z_{3}}{z_{1} z_{2}}-1\right) \theta_{1} \theta_{2}+\mu\left(\frac{z_{1} z_{2}}{z_{3}}-1\right) \theta_{3} .
$$

Performing the substitutions $\theta_{1} \mapsto a-\theta_{3}, \theta_{2} \mapsto b-\theta_{3}, z_{1} \mapsto 1$ and $z_{2} \mapsto 1$, leads, to the reduced Hamiltonian $H^{\prime}=\lambda\left(z_{3}-1\right)\left(b-\theta_{3}\right)\left(b-\theta_{3}\right)+\mu\left(z_{3}{ }^{-1}-1\right) \theta_{3}$, which is an element of $\operatorname{Weyl}_{\mathbb{R}}\left(z_{3}\right)$. The bound $N_{3}(t) \leq m$ with $m=\min (a, b)$ implies that the function $f\left(\nu_{3}\right)=\nu_{3}\left(\nu_{3}-1\right) \cdots\left(\nu_{3}-m\right)$ is zero at all time $t$. One then gets

$$
f\left(\theta_{3}\right) \phi\left(t, z_{3}\right)=\sum_{0 \leq \nu_{3} \leq m} \pi_{\nu_{3}}(t) f\left(\nu_{3}\right) z_{3}^{\nu_{3}}=0, \quad \forall t \in \mathbb{R}_{\geq 0} .
$$

The operator $f\left(\theta_{3}\right)$ vanishes on the generating series $\phi\left(t, z_{3}\right)$. All computations can therefore be done in the quotient of the algebra $\operatorname{Weyl}_{\mathbb{R}}\left(z_{3}\right)$ by the left ideal generated by $f\left(\theta_{3}\right)$. From an algorithmic point of view, this can be achieved by Gröbner basis techniques on the polynomials $f_{\kappa}$ (which are commutative polynomials in $\theta$ ).

\section{A combined formula for differentiating and evaluating}

In this section, the explicit Formula (14) is given for computing $f_{\kappa}$ (Formula 12) which are commutators evaluated at $z=1$. We believe that this formula has several advantages. First, this combined formula provides an improvement of the algorithm of Section 4. Indeed, Formula (14) can be implemented only with basic operations on commutative polynomials in $\theta$. Moreover, Formula (14) does not 
need any specialization $z=1$. Second, all computations in the Weyl algebra can be avoided, which makes the algorithm easier to implement since no library for computing in Weyl algebras is needed. Indeed, Weyl algebras computations are, to our knowledge, only available through softwares like Maple [2, 1], Macaulay 2 [10], which makes it hard to produce an independant or GPL standalone library. Third, we believe that Formula (14) helps finding interesting formulas such as in Lemma 9 (page 19). Fourth, the computation of Formula (14) can be easily mixed with the reductions detailed in section 5 by using modular exponentiations.

Lemma 5. Let $A$ and $B$ taken in $\operatorname{Weyl}_{\mathbb{R}}\left(z_{1}, \ldots, z_{n}\right)$. Define $\operatorname{ad}_{A}(B)=[A, B]$. Then

$$
A^{k} B=\sum_{i=0}^{k}\left(\begin{array}{l}
k \\
i
\end{array}\right) \operatorname{ad}_{A}^{i}(B) A^{k-i}
$$

where $\operatorname{ad}_{A}^{i}$ denotes the $\operatorname{ad}_{A}$ function composed $i$ times.

Proof. Let $t$ be an indeterminate. The classical identity, between formal series in $t, \exp (t A) B \exp (-t A)=\exp \left(t \operatorname{ad}_{A}\right)(B)$ holds (adjoint representation of a Lie group over its Lie algebra, see [3] for details). This formula can be rewritten as $\exp (t A) B=\exp \left(t \operatorname{ad}_{A}\right)(B) \exp (t A)$ and developed with a Taylor expansion. The result is proved by identifying the coefficients of $t^{k}$ in each side of

$$
\sum_{k \geq 0} \frac{t^{k}}{k !} A^{k} B=\sum_{i, j \geq 0} \frac{t^{i+j}}{i ! j !} \operatorname{ad}_{A}^{i}(B) A^{j}
$$

Lemma 6. For all $m \in \mathbb{Z}$ and any $k \in \mathbb{N}$, one has $\theta^{k} z^{m}=z^{m}(m+\theta)^{k}$ with $\theta=z \frac{\partial}{\partial z}$.

Proof. One has $\operatorname{ad}_{\theta}\left(z^{m}\right)=\left[\theta, z^{m}\right]=m z^{m}$. Thus, $\operatorname{ad}_{\theta}^{i}\left(z^{m}\right)=m^{i} z^{m}$ for any $i \geq 0$. The lemma then follows from Lemma 5 by taking $A=\theta$ and $B=z^{m}$.

Let $\nu \in \mathbb{Z}^{n}$ and $\kappa \in \mathbb{N}^{n}$. Denote $(\nu+\theta)^{\kappa}=\left(\nu_{1}+\theta_{1}\right)^{\kappa_{1}}\left(\nu_{2}+\theta_{2}\right)^{\kappa_{2}} \cdots\left(\nu_{n}+\theta_{n}\right)^{\kappa_{n}}$.

Lemma 7. The commutation relation between $\theta^{\kappa}$ and $z^{\nu}$, viewed as an element of $\operatorname{Weyl}_{\mathbb{R}}\left(z_{1}, z_{2}, \ldots, z_{n}\right)$, can now be written:

$$
\theta^{\kappa} z^{\nu}=z^{\nu}(\nu+\theta)^{\kappa}
$$

Proof. The proof relies on Lemma 6. It is only given for $n=3$.

$$
\begin{aligned}
\theta^{\kappa} z^{\nu} & =\left(\theta_{1}^{\kappa_{1}} \theta_{2}^{\kappa_{2}} \theta_{3}^{\kappa_{3}}\right)\left(z_{1}^{\nu_{1}} z_{2}^{\nu_{2}} z_{3}^{\nu_{3}}\right)=\left(\theta_{1}^{\kappa_{1}} z_{1}^{\nu_{1}}\right)\left(\theta_{2}^{\kappa_{2}} z_{2}^{\nu_{2}}\right)\left(\theta_{3}^{\kappa_{3}} z_{3}^{\nu_{3}}\right) \\
& =z_{1}^{\nu_{1}}\left(\nu_{1}+\theta_{1}\right)^{\kappa_{1}} z_{2}^{\nu_{2}}\left(\nu_{2}+\theta_{2}\right)^{\kappa_{2}} z_{3}^{\nu_{3}}\left(\nu_{3}+\theta_{3}\right)^{\kappa_{3}} \\
& =\left(z_{1}^{\nu_{1}} z_{2}^{\nu_{2}} z_{3}^{\nu_{3}}\right)\left(\nu_{1}+\theta_{1}\right)^{\kappa_{1}}\left(\nu_{2}+\theta_{2}\right)^{\kappa_{2}}\left(\nu_{3}+\theta_{3}\right)^{\kappa_{3}}=z^{\nu}(\nu+\theta)^{\kappa} .
\end{aligned}
$$

Proposition 2. Let $\mathcal{R}$ be a stochastic Petri net, the Hamiltonian of which is $H$. Then

$$
\left[\theta^{\kappa}, H\right]_{\left.\right|_{z=1}}=\sum_{(c, \alpha, \beta) \in \mathcal{R}} \frac{c}{\alpha !}\left[(\beta-\alpha+\theta)^{\kappa}-\theta^{\kappa}\right] \theta^{\underline{\alpha}}, \quad\left(\kappa \in \mathbb{N}^{n}\right) .
$$


Proof. The evaluated commutator $\left[\theta^{\kappa}, H\right]_{\left.\right|_{z=1}}$ is linear in $H$. For simplicity, it is assumed that a single chemical reaction is involved, so that $H=\left(z^{\beta-\alpha}-1\right) \theta^{\underline{\alpha}}$. The proof reduces to the computation of $\left[\theta^{\kappa},\left(z^{\beta-\alpha}-1\right) \theta^{\underline{\alpha}}\right]_{\left.\right|_{z=1}}$. Lemma 7 is used to make the terms $\theta^{\kappa}$ and $z^{\beta-\alpha}-1$ commute. Computations give the following formula

$$
\theta^{\kappa}\left(z^{\beta-\alpha}-1\right)=\theta^{\kappa} z^{\beta-\alpha}-\theta^{\kappa}=z^{\beta-\alpha}(\beta-\alpha+\theta)^{\kappa}-\theta^{\kappa}
$$

Evaluate it at $z=1$ and apply the fact that $H_{\left.\right|_{z=1}}=0$. The proposition is proved.

\section{Order of a Chemical Reaction System}

In this section, well-known results on order 1 systems are recovered from the Weyl algebra theory (see [12] and [4, sect. 5.3.3]). First, some further theoretical developments on Weyl algebra are introduced.

The algebra $\operatorname{Weyl}_{\mathbb{R}}(z)=\operatorname{Weyl}_{\mathbb{R}}\left(z_{1}, \ldots, z_{n}\right)$ is graded by the degree. It is readily checked that the product $A B$ of two elements $A, B \in \operatorname{Weyl}_{\mathbb{R}}(z)$ homogeneous by degree, is also homogeneous of degree $\operatorname{deg}(A)+\operatorname{deg}(B)$. Moreover, the algebra is filtered by the order (in the sense of differential operators). The component $\mathcal{F}_{q} \subset \operatorname{Weyl}_{\mathbb{R}}(z), q \in \mathbb{N}$, of the growing filtration

$$
\mathcal{F}: \mathcal{F}_{0} \subset \mathcal{F}_{1} \subset \mathcal{F}_{2} \subset \cdots
$$

is the $\mathbb{R}$-vector space spanned by the elements $z^{\alpha} \partial_{z}^{\beta}$ of order at most $q$, i.e. such that $|\beta| \leq q$. It is possible to check that $\mathcal{F}_{k} \mathcal{F}_{l} \subset \mathcal{F}_{k+l}$ for all $k, l \in \mathbb{N}$. The graded algebra associated to the filtration $\mathcal{F}$ is commutative since $\left[\mathcal{F}_{k}, \mathcal{F}_{l}\right] \subset \mathcal{F}_{k+l-1}$ for all $k, l \in \mathbb{N}$ (with the convention $\mathcal{F}_{-1}=\{0\}$ ). It is defined by

$$
\operatorname{gr} \operatorname{Weyl}_{\mathbb{R}}(z)=\mathcal{F}_{0} \oplus \mathcal{F}_{1} / \mathcal{F}_{0} \oplus \mathcal{F}_{2} / \mathcal{F}_{1} \oplus \cdots
$$

Definition 6. A chemical reaction system $\mathcal{R}$ is said to be of order $q \in \mathbb{N}$, if each reaction $(c, \alpha, \beta) \in \mathcal{R}$ satisfies $|\alpha| \leq q$ (where $|\alpha|=\alpha_{1}+\alpha_{2}+\cdots+\alpha_{n}$ for any $\left.\alpha \in \mathbb{N}^{n}\right)$.

Thus, a chemical reaction system $\mathcal{R}$ is of order $q$ if every reaction of $\mathcal{R}$ consumes at most $q$ molecules. The next lemma follows immediately from Formula (11).

Lemma 8. A chemical reaction system is of order $q$ iff its Hamiltonian $H$ belongs to the $\mathcal{F}_{q}$ component of the Weyl algebra filtration defined by (15).

Any polynomial $f \in \mathbb{R}\left[\theta_{1}, \theta_{2}, \ldots, \theta_{n}\right]$, homogeneous of degree $d$, defines a degree $d$ moment $\operatorname{E} f(N(t))$.

Proposition 3. In a chemical reaction system of order $q$, the derivative with respect to the time of a degree $d$ moment, only depends on other moments of order at most $q+d-1$. 
Proof. Let $H \in \operatorname{Weyl}_{\mathbb{R}}\left(z_{1}, z_{2}, \ldots, z_{n}\right)$ be an order $q$ operator i.e. $H \in \mathcal{F}_{q}$. If $f \in \mathbb{R}\left[\theta_{1}, \theta_{2}, \ldots, \theta_{n}\right]$ is an homogeneous polynomial of degree $d$, then one has $[f(\theta), H] \in \mathcal{F}_{q+d-1}$. Evaluation at $z=1$ yields $[f(\theta), H]_{\mid z=1} \in \mathbb{R}\left[\theta_{1}, \theta_{2}, \ldots, \theta_{n}\right]$ of degree at most $q+d-1$.

Corollary 1. In a chemical reaction system, the dynamics of the mean values E $N_{k}(t), k=1 \ldots n$, (i.e. first order moments) can be written as follows (with $\left.k \in \mathbb{N}, \alpha, \beta \in \mathbb{N}^{n}\right)$. Moreover, the dynamics of a first order system is linear in the variables $\mathrm{E} N_{k}(t)$.

$$
\frac{d}{d t} \mathrm{E} N_{k}(t)=\sum_{(c, \alpha, \beta) \in \mathcal{R}} \frac{c}{\alpha !}\left(\beta_{k}-\alpha_{k}\right) \mathrm{E} N(t)^{\underline{\alpha}}
$$

Proof. One deduces $\theta_{k} H=\sum_{(c, \alpha, \beta) \in \mathcal{R}} \frac{c}{\alpha !}\left(\beta_{k}-\alpha_{k}\right) z^{\beta-\alpha} \theta^{\underline{\alpha}}+\frac{c}{\alpha !}\left(z^{\beta-\alpha}-1\right) \theta_{k} \theta^{\underline{\alpha}}$ from Formula (11). The evaluation at the point $z=1$ leads to the relation $\theta_{k} H_{\left.\right|_{z=1}}=\sum_{(c, \alpha, \beta) \in \mathcal{R}} \frac{c}{\alpha !}\left(\beta_{k}-\alpha_{k}\right) \theta^{\underline{\alpha}}$. Applying Lemma 2 and Proposition 1, the formula is proved. Whenever $|\alpha| \leq 1, \alpha !=1$ and $N(t)^{\underline{\alpha}}=N(t)^{\alpha}$. Moreover $N(t)^{\alpha}=1$ iff $\alpha=0$ and $N(t)^{\alpha}=N_{j}(t)$ if $\alpha=(0, \ldots, 0,1,0, \ldots, 0)$, the " 1 " occuring in $j^{\text {th }}$ position.

The deterministic models in chemical kinetics, classically built using the mass action law, are recovered. For first order systems, the deterministic model corresponds to an unbiased averaging of the random variables $N_{k}(t)$. It does not apply to covariance matrices $\operatorname{Cov}\left(N_{i}(t), N_{j}(t)\right)$, where $i, j=1 \ldots n$.

\section{Examples}

\subsection{First Order Systems}

The following model of a non-regulated gene [11] is interesting because it was verified experimentally and because it shows the importance of random phenomena in gene expression. The transcription of the gene produces messenger RNAs (mRNA) which are translated into proteins:

$$
\begin{cases}\text { DNA } \stackrel{k_{R}}{\longrightarrow} \text { DNA + mRNA } & \text { (transcription) } \\ \text { mRNA } \stackrel{k_{P}}{\longrightarrow} \text { mRNA + protein } & \text { (translation) } \\ \text { mRNA } \stackrel{\gamma_{R}}{\longrightarrow} \emptyset & \text { (mRNA degradation) } \\ \text { protein } \stackrel{\gamma_{P}}{\longrightarrow} \emptyset & \text { (protein degradation) }\end{cases}
$$

The three chemical species are numbered is the following manner: $R_{1}=$ DNA, $R_{2}=$ mRNA and $R_{3}=$ protein. It admits the conservation law $\nu_{1}=1$, meaning that there is only one gene involved in the network (at any time $t$ ). The model is reduced by setting $\theta_{1}=1$ and $z_{1}=1$. The reduced Hamiltonian is equal to

$$
H=k_{R}\left(z_{2}-1\right)+k_{P}\left(z_{3}-1\right) \theta_{2}+\gamma_{R}\left(z_{2}^{-1}-1\right) \theta_{2}+\gamma_{P}\left(z_{3}^{-1}-1\right) \theta_{3}
$$


Only two state variables remain $\nu=\left(\nu_{2}, \nu_{3}\right) \in \mathbb{N}^{2}$, the number of mRNA and the number of proteins at time $t$. Let $N(t)=\left(N_{2}(t), N_{3}(t)\right)$ be the stochastic process associated to (17). We are going to compute the means $x_{2}(t)=\mathrm{E} N_{2}(t)$ et $x_{3}(t)=\mathrm{E} N_{3}(t)$ and the covariance matrix $x_{i j}(t)=\operatorname{Cov}\left(N_{i}(t), N_{j}(t)\right)$ for $i, j=2,3$. According to the algorithm of Section 4, our software computes the commutators evaluated at the point $z_{2}=z_{3}=1$ :

$$
\begin{aligned}
{\left[\theta_{2}, H\right]_{\left.\right|_{z=1}} } & =k_{R}-\gamma_{R} \theta_{2} \\
{\left[\theta_{3}, H\right]_{\left.\right|_{z=1}} } & =k_{P} \theta_{2}-\gamma_{P} \theta_{3} \\
{\left[\theta_{2}^{2}, H\right]_{\left.\right|_{z=1}} } & =k_{R}+\left(2 k_{R}+\gamma_{R}\right) \theta_{2}-2 \gamma_{R} \theta_{2}^{2} \\
{\left[\theta_{2} \theta_{3}, H\right]_{\left.\right|_{z=1}} } & =k_{R} \theta_{3}+k_{P} \theta_{2}^{2}+\left(-\gamma_{R}-\gamma_{P}\right) \theta_{2} \theta_{3} \\
{\left[\theta_{3}^{2}, H\right]_{\left.\right|_{z=1}} } & =k_{P} \theta_{2}+\gamma_{P} \theta_{3}+2 k_{P} \theta_{2} \theta_{3}-2 \gamma_{P} \theta_{3}^{2}
\end{aligned}
$$

and generates the system which describes the time evolution of the means and the covariances:

$$
\begin{aligned}
\frac{d}{d t} x_{2}(t) & =k_{R}-\gamma_{R} x_{2}(t) \\
\frac{d}{d t} x_{3}(t) & =k_{P} x_{2}(t)-\gamma_{P} x_{3}(t) \\
\frac{d}{d t} x_{2,2}(t) & =\gamma_{R} x_{2}(t)+k_{R}-2 \gamma_{R} x_{2,2}(t) \\
\frac{d}{d t} x_{2,3}(t) & =\left(-\gamma_{R}-\gamma_{P}\right) x_{2,3}(t)+k_{P} x_{2,2}(t) \\
\frac{d}{d t} x_{3,3}(t) & =k_{P} x_{2}(t)-2 \gamma_{P} x_{3,3}(t)+2 k_{P} x_{2,3}(t)+\gamma_{P} x_{3}(t)
\end{aligned}
$$

Since the chemical reaction system has order 1 , a linear system is obtained and the phenomenon of the infinite cascade does not occur. For simplification, a time scale such that $\gamma_{R}=1$ is selected. The computation of the means and the variances at the stationary state give:

$$
\begin{gathered}
x_{2}=k_{R}, \quad x_{3}=\frac{k_{P} k_{R}}{\gamma_{P}}, \\
x_{2,2}=k_{R}, \quad x_{2,3}=\frac{k_{P} k_{R}}{1+\gamma_{P}}, \quad x_{3,3}=\frac{k_{P} k_{R}\left(\gamma_{P}+k_{P}+1\right)}{\gamma_{P}\left(1+\gamma_{P}\right)}
\end{gathered}
$$

This result is exact. The same formulas appear in [11] and are proved by using Langevin's technique: the two first equations of (18) are viewed as a deterministic model (arising from the mass action law). Then, two white noises of zero average are incorporated in the righthand sides. This method is difficult to justify theoretically. By solving the equations (18), by, say, a Laplace transform technique, we obtain exact formulas for the means and the variances during the 
transient stage, given below:

$$
\begin{aligned}
x_{2}(t) & =k_{R}\left(-\mathrm{e}^{-t}+1\right) \\
x_{3}(t) & =\frac{k_{P} k_{R}\left(-1+\mathrm{e}^{-\gamma_{P} t}+\gamma_{P}\left(-\mathrm{e}^{-t}+1\right)\right)}{\gamma_{P}\left(-1+\gamma_{P}\right)} \\
x_{2,2}(t) & =k_{R}\left(-\mathrm{e}^{-t}+1\right) \\
x_{2,3}(t) & =\frac{\left(-\mathrm{e}^{-t}\left(1+\gamma_{P}\right)+\gamma_{P}+\mathrm{e}^{-\left(1+\gamma_{P}\right) t}\right) k_{R} k_{P}}{\gamma_{P}\left(1+\gamma_{P}\right)}
\end{aligned}
$$

\subsection{Second Order Systems}

The dynamics of the degree $d$ moments depends on moments of degree strictly greater than $d$. In other words, an infinite cascade occurs. This is the main source of difficulty. In order to break an infinite cascade there are two possible methods:

1. One can operate an approximation assuming that the centered moments of degree $d$ are zero for $d$ large enough. This is a legitimate approximation whenever the number of tokens in each place (the number of chemical molecules of each chemical species) remains high at any time $t$.

2. One has a relation expressing degree $d+1$ moments as a function of moments of degree at most $d$. This case occurs, in particular, whenever random variables only take a finite number of different values.

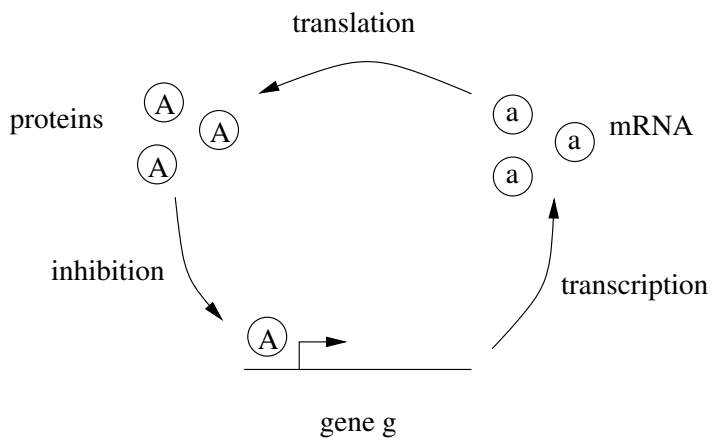

Fig. 3. Autoregulated gene

Autoregulated Gene The transcription of the gene produces messenger RNA, which in turn are translated in proteins. If a protein binds to the gene, then the 
transcription is blocked. (see Figure 3).

$$
\begin{aligned}
& \text { gene } \stackrel{\lambda_{1}}{\longrightarrow} \text { gene }+ \text { mRNA } \\
& \text { mRNA } \stackrel{\lambda_{2}}{\longrightarrow} \text { mRNA + protein } \\
& \operatorname{mRNA} \stackrel{\mu_{1}}{\longrightarrow} \emptyset \\
& \text { protein } \stackrel{\mu_{2}}{\longrightarrow} \emptyset \\
& \text { gene }+ \text { protein } \stackrel{c_{1}}{\longrightarrow} \text { blocked_gene } \\
& \text { blocked_gene } \stackrel{c_{2}}{\longrightarrow} \text { gene }+ \text { protein }
\end{aligned}
$$

The four chemical species are numbered: $R_{1}=$ mRNA, $R_{2}=$ gene, $R_{3}=$ blocked gene, $R_{4}=$ protein. That system obeys the conservation law $I(\nu)=\nu_{2}+\nu_{3}$, meaning that the total number of "molecules" of type gene and blocked_gene remains constant. In practice, only one gene is involved. Therefore the Petri net gets initialized with the following assumption $\nu_{2}+\nu_{3}=1$. Since at any time $t$, $\nu_{2}+\nu_{3}=1$, we choose to remove the state variable $\nu_{3}$ putting $\nu_{3}=1-\nu_{2}$. After model reduction, the Hamiltonian becomes

$$
\begin{aligned}
H= & \lambda_{1}\left(z_{1}-1\right) \theta_{2}+\lambda_{2}\left(z_{4}-1\right) \theta_{1}+\mu_{1}\left(\frac{1}{z_{1}}-1\right) \theta_{1}+\mu_{2}\left(\frac{1}{z_{4}}-1\right) \theta_{4} \\
& +c_{1}\left(\frac{1}{z_{2} z_{4}}-1\right) \theta_{2} \theta_{4}+c_{2}\left(z_{2} z_{4}-1\right)\left(1-\theta_{2}\right) .
\end{aligned}
$$

We introduce the means $x_{i}(t)=\mathrm{E} N_{i}(t)$ for $i=1,2,4$ and the covariance matrix $x_{i j}(t)=\operatorname{Cov}\left(N_{i}(t), N_{j}(t)\right)$ for $i, j=1,2,4$. The random variable $N_{2}(t)$ is boolean $\left(\nu_{2}=\nu_{2}^{2}\right)$, the model restriction algorithm can therefore be applied. The algorithm computes the brackets $\left[\theta^{\kappa}, H\right]$ in the Weyl algebra quotiented by the left ideal spanned by the relation $\theta_{2}=\theta_{2}^{2}$. The evaluation at $z=1$ is performed afterwards. It yields:

$$
\begin{aligned}
{\left[\theta_{1}, H\right]_{\left.\right|_{z=1}} } & =-\mu_{1} \theta_{1}+\lambda_{1} \theta_{2} \\
{\left[\theta_{2}, H\right]_{\left.\right|_{z=1}} } & =c_{2}-c_{2} \theta_{2}-c_{1} \theta_{2} \theta_{4} \\
{\left[\theta_{4}, H\right]_{\left.\right|_{z=1}} } & =c_{2}+\lambda_{2} \theta_{1}-c_{2} \theta_{2}-\mu_{2} \theta_{4}-c_{1} \theta_{2} \theta_{4} \\
{\left[\theta_{1}^{2}, H\right]_{\left.\right|_{z=1}} } & =\mu_{1} \theta_{1}+\lambda_{1} \theta_{2}-2 \mu_{1} \theta_{1}^{2}+2 \lambda_{1} \theta_{1} \theta_{2} \\
{\left[\theta_{2} \theta_{1}, H\right]_{\left.\right|_{z=1}} } & =c_{2} \theta_{1}+\lambda_{1} \theta_{2}-\left(\mu_{1}+c_{2}\right) \theta_{1} \theta_{2}-c_{1} \theta_{1} \theta_{2} \theta_{4}
\end{aligned}
$$

An infinite cascade occurs because the dynamics on the degree 2 moments involves two degree three moments, namely the moment coded by the following operators $\theta_{1} \theta_{2} \theta_{4}$ and $\theta_{2} \theta_{4}^{2}$. The approximation assumption that all centered moments of degree 3 are zero, can be used to break this cascade. Consider three random variables $\left(X_{1}, X_{2}, X_{3}\right)$ with respective means $\left(x_{1}, x_{2}, x_{3}\right)$. A routine computation gives the centered moment of order three $\mathrm{E}\left(\left(X_{1}-x_{1}\right)\left(X_{2}-x_{2}\right)\left(X_{3}-x_{3}\right)\right)$ as $\mathrm{E}\left(X_{1} X_{2} X_{3}\right)-x_{1} x_{2} x_{3}-x_{1} \operatorname{Cov}\left(X_{2}, X_{3}\right)-x_{2} \operatorname{Cov}\left(X_{3}, X_{1}\right)-x_{3} \operatorname{Cov}\left(X_{1}, X_{2}\right)$. We have no proof that this approximation is best fit. Our software computes the 
ordinary non-linear differential equation system:

$$
\begin{aligned}
\frac{d}{d t} x_{1}(t)= & -\mu_{1} x_{1}(t)+\lambda_{1} x_{2}(t) \\
\frac{d}{d t} x_{2}(t)= & c_{2}-c_{2} x_{2}(t)-c_{1} x_{4}(t) x_{2}(t)-c_{1} x_{2,4}(t) \\
\frac{d}{d t} x_{4}(t)= & c_{2}+\lambda_{2} x_{1}(t)-c_{2} x_{2}(t)-c_{1} x_{4}(t) x_{2}(t) \\
& -\mu_{2} x_{4}(t)-c_{1} x_{2,4}(t) \\
\frac{d}{d t} x_{1,1}(t)= & \mu_{1} x_{1}(t)+2 \lambda_{1} x_{1,2}(t)+\lambda_{1} x_{2}(t)-2 \mu_{1} x_{1,1}(t) \\
\frac{d}{d t} x_{1,2}(t)= & \lambda_{1} x_{2}(t)-c_{1} x_{1,4}(t) x_{2}(t)-c_{1} x_{4}(t) x_{1,2}(t) \\
& -\left(\mu_{1}+c_{2}\right) x_{1,2}(t)-\lambda_{1} x_{2}(t)^{2}
\end{aligned}
$$

The Figures 4, 5 and 6 show the numerical simulations produced by our software. We used the parameter values $\lambda_{1}=30.0, \lambda_{2}=10.0, \mu_{1}=\mu_{2}=0.1$, and $c_{1}=c_{2}=1.0$, and the initial conditions $x_{1}(0)=0(\mathrm{mRNA}), x_{2}(0)=1$ (gene), $x_{3}(0)=0$ (protein).

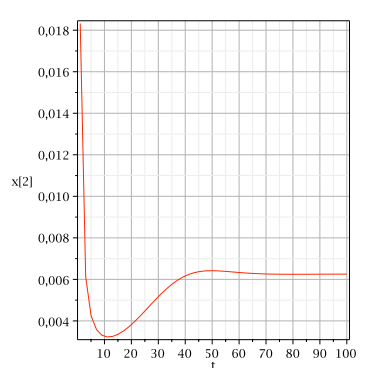

(a) Average expression rate of the gene

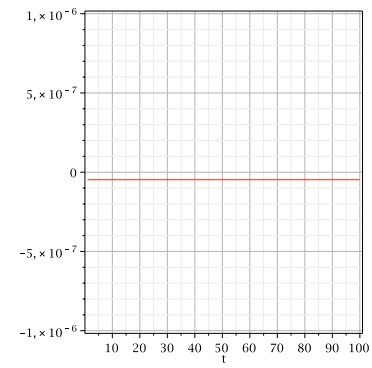

(b) Accuracy test on variance

Fig. 4. Autoregulated gene (boolean variable). Simulations are consistent with the fact that any boolean variable $X$ satifies $\operatorname{Var} X=x(1-x)$, where $x=\mathrm{E} X$.

Second Order Degradation Consider the chemical reaction

$$
2 R \stackrel{\mu}{\longrightarrow} \emptyset
$$

Using a time dilatation, it is assumed that $\mu=1$. The Hamiltonian is then, with $\theta=z \partial / \partial z$ :

$$
H=\frac{1}{2}\left(\frac{1}{z^{2}}-1\right) \theta(\theta-1)=\frac{1}{2}\left(1-z^{2}\right)\left(\frac{\partial}{\partial z}\right)^{2}
$$




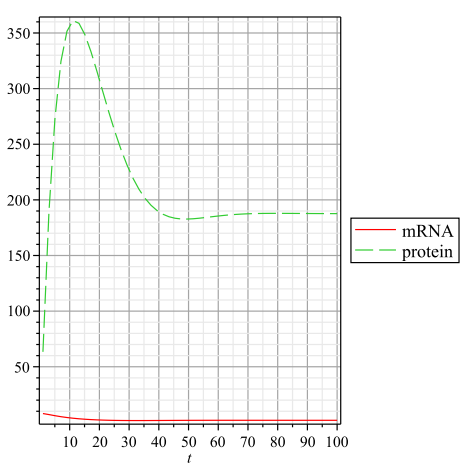

(a) Average number of mRNA and proteins

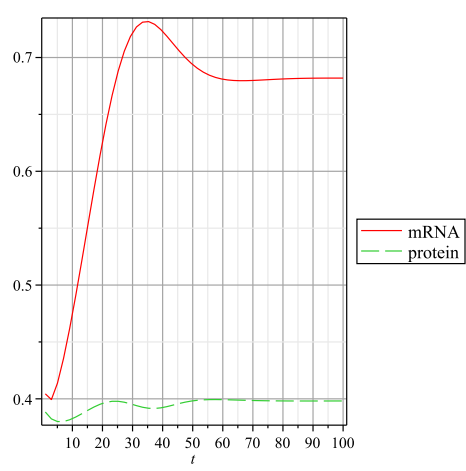

(b) Relative standard deviation

Fig. 5. Joint evolution of mRNA and protein number. The relative standard deviation of a random variable $X$ is $\sigma / x$ with $\sigma^{2}:=\operatorname{Var} X$ and $x:=\mathrm{E}(X)$.

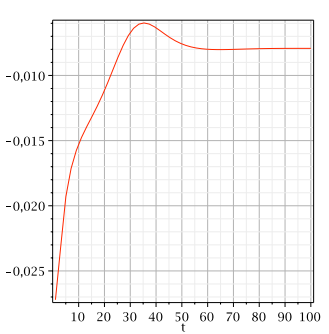

(a) Gene-mRNA correlation

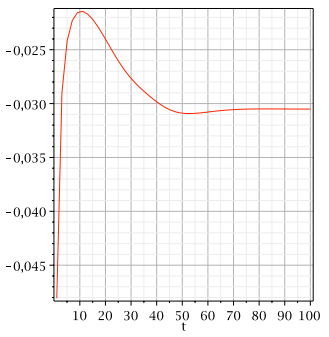

(b) Gene-protein correlation

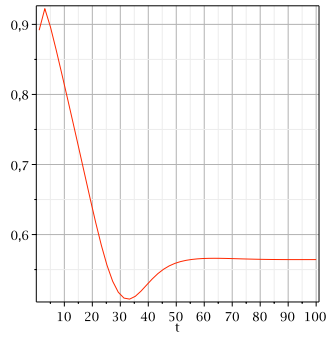

(c) mRNA-protein correlation

Fig. 6. Evolution over time of correlation rates. The correlation rate $c(X, Y)$ between two random variables $X$ and $Y$ is defined by $c(X, Y):=\frac{\operatorname{Cov}(X, Y)}{\sigma(X) \sigma(Y)}$.

On this example, the dynamics on the moments $\mathrm{E} N(t)^{k}, k=1 \ldots 4$ is coded by

$$
\begin{aligned}
{[\theta, H]_{\left.\right|_{z=1}} } & =-\theta^{2}+\theta \\
{\left[\theta^{2}, H\right]_{\left.\right|_{z=1}} } & =-2 \theta^{3}+4 \theta^{2}-2 \theta \\
{\left[\theta^{3}, H\right]_{\left.\right|_{z=1}} } & =-3 \theta^{4}+9 \theta^{3}-10 \theta^{2}+4 \theta \\
{\left[\theta^{4}, H\right]_{\left.\right|_{z=1}} } & =-4 \theta^{5}+16 \theta^{4}-28 \theta^{3}+24 \theta^{2}-8 \theta
\end{aligned}
$$

Lemma 9. For all $m \in \mathbb{N}$, Formulas (20) take the following closed form:

$$
\left[\theta^{m}, H\right]_{\left.\right|_{z=1}}=\frac{1}{2}\left[(-2+\theta)^{m}-\theta^{m}\right] \theta(\theta-1)
$$

Proof. Apply Formula (14) for $(c, \alpha, \beta):=(1,(2),(0))$. 
Let $x(t)$ denote the mean and $v(t)$ the variance of $N(t)$. The previously developped approximation, obtained by killing centered order 3 moments, then gives

$$
\begin{aligned}
& \frac{d}{d t} x(t)=x(t)-v(t)-(x(t))^{2} \\
& \frac{d}{d t} v(t)=-2 x(t)+4 v(t)+2(x(t))^{2}-4 v(t) x(t) .
\end{aligned}
$$

This approximation method behaves badly when E $N(t)$ gets smaller than 1 (see Figure 7). Unfortunately the situation does not improve if the centered moment is kept up to a higher order.

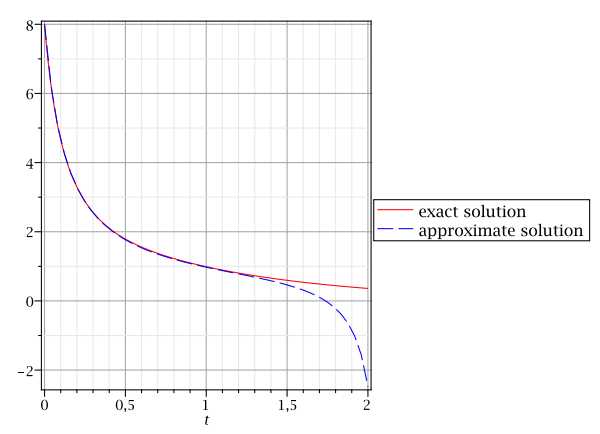

Fig. 7. Evolution of the mean $x(t)=\mathrm{E} N(t)$ over time, starting with state $n_{0}=8$. The approximation gets worse after $x(t)$ crosses the value 1 (around $t=1$ ).

We show now how to get the exact dynamics on the moments of any order. Assume, as an example, that the initial state is $n_{0}=8$. Then, the polynomial function $f(\nu)=\nu(\nu-2)(\nu-4)(\nu-6)(\nu-8)$ is zero at any instant $t$. According to Lemma 2 and Proposition 1, we have to quotient the algebra $\operatorname{Weyl}_{\mathbb{R}}(z)$ by the left ideal spanned by the relation $f(\theta)=0$. This leads to $f(\theta)=\theta(\theta-2)(\theta-4)(\theta-6)(\theta-8)$. Adding this extra relation to System $(20)$ enables us to get order 5 moments as functions of the moments of order at most 4. This way, we get the exact dynamics, a linear system, describing the time evolution of the moments $x_{k}(t)=\mathrm{E} N^{k}(t)$, for $k=1 \ldots 4$.

$$
\begin{aligned}
& \frac{d}{d t} x_{1}(t)=x_{1}(t)-x_{2}(t) \\
& \frac{d}{d t} x_{2}(t)=-2 x_{1}(t)+4 x_{2}(t)-2 x_{3}(t) \\
& \frac{d}{d t} x_{3}(t)=4 x_{1}(t)-10 x_{2}(t)+9 x_{3}(t)-3 x_{4}(t) \\
& \frac{d}{d t} x_{4}(t)=1528 x_{1}(t)-1576 x_{2}(t)+532 x_{3}(t)-64 x_{4}(t)
\end{aligned}
$$


with the initial conditions: $x_{1}(0)=8, x_{2}(0)=64, x_{3}(0)=512, x_{4}(0)=4096$. These linear differential equations get solved by means of the Laplace transform:

$$
\begin{aligned}
& x_{1}(t)=\frac{8}{3} \mathrm{e}^{-t}+\frac{112}{33} \mathrm{e}^{-6 t}+\frac{64}{39} \mathrm{e}^{-15 t}+\frac{128}{429} \mathrm{e}^{-28 t} \\
& x_{2}(t)=\frac{16}{3} \mathrm{e}^{-t}+\frac{784}{33} \mathrm{e}^{-6 t}+\frac{1024}{39} \mathrm{e}^{-15 t}+\frac{3712}{429} \mathrm{e}^{-28 t} \\
& x_{3}(t)=\cdots
\end{aligned}
$$

The infinite cascade if thereby broken by an exact method (see the simulations shown in Figure 8)

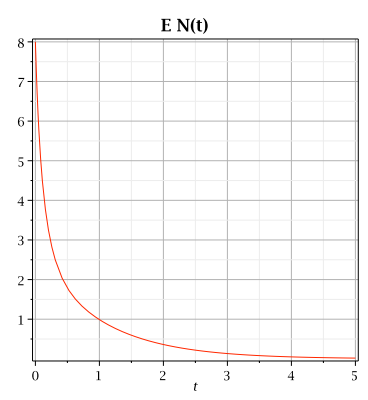

(a) Mean $x(t)$

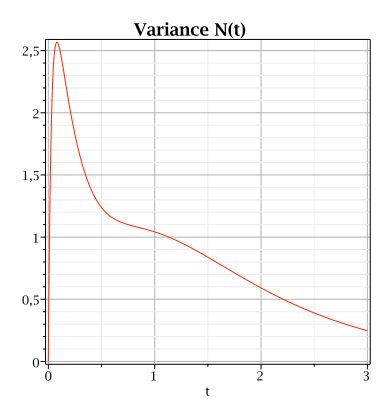

(b) Variance $v(t)$

Fig. 8. Simulation of System (19), starting with state $n_{0}=8$.

\section{Conclusion}

The algorithm presented in this paper allows the investigation on the study of genetic regulatory networks, considered from a stochastic point of view. The algorithm yields a system of differential equations whose integration yields values for some moments of the number of molecules of chemical species. The algorithm and its theory are formulated in the Weyl algebra. However, Proposition 2 shows how to replace computations in Weyl algebra by basic operations on commutative polynomials. This seems to produce a more efficient algorithm which combines the differentiation and evaluation steps of the straightforward approach. The issue of the infinite cascade, well-known is statistical physics, reduces the usefulness of the overall method. Approximation techniques, useful to break it, still need some investigation. 


\section{References}

1. S. A. Abramov, H. Q. Le, and Z. Li. OreTools: a computer algebra library for univariate ore polynomial rings. School of Computer Science CS-2003-12, University of Waterloo, 2003.

2. Frédéric Chyzak. The Ore_algebra library. In Maple, Maplesoft, Canada. Software.

3. J. Dixmier. Enveloping Algebras. American Mathematical Society, 1996. (Translation of the french edition Algèbres enveloppantes published in 1974 by Bordas).

4. Péter Érdi and János Tóth. Mathematical models of chemical reactions. Princeton University Press, 1989.

5. William Feller. An introduction to probability theory and its applications. Vol. I. John Wiley and Sons, Inc., New York, 1957. 2nd ed.

6. C.S. Gillespie. Moment-closure approximations for mass-action models. Systems Biology, IET, 3(1):52 -58, January 2009.

7. Daniel T. Gillespie. Exact Stochastic Simulation of Coupled Chemical Reactions. Journal of Physical Chemistry, 81(25):2340-2361, 1977.

8. A. V. Kalinkin. Markov branching processes with interaction. Russian Math. Surveys, 57:241-304, 2002.

9. Steffen Klamt and Jörg Stelling. Stoichiometric and Constraint-based Modeling. In Zoltan Szallasi, Jörg Stelling, and Vipul Periwal, editors, System Modeling in Cellular Biology: From Concepts to Nuts and Bolts, pages 73-96. Cambridge, Massachussets: The MIT Press, 2006.

10. A. Leykin. D-modules for macaulay 2. mathematical software. In World Sci. Publishing, editor, Mathematical software, pages 169-179, River Edge, 2002. (Beijing, 2002).

11. M. Ozbudak, M. Thattai, Iren Kurtser, and Alan D. Grossman. Regulation of noise in the expression of a single gene. Nature genetics, 31:69-73, May 2002.

12. Johan Paulsson. Models of stochastic gene expression. Physics of live rev., 2:157175, May 2005.

13. Johan Paulsson and Johan Elf. Stochastic Modeling of Intracellular Kinetics. In Zoltan Szallasi, Jörg Stelling, and Vipul Periwal, editors, System Modeling in Cellular Biology: From Concepts to Nuts and Bolts, pages 149-175. Cambridge, Massachussets: The MIT Press, 2006.

14. Christophe Reutenauer. Aspects mathématiques des réseaux de Petri. Masson, 1989.

15. Samuel Alexandre Vidal. Groupe Modulaire et Cartes Combinatoires. Génération et Comptage. PhD thesis, Université Lille I, France, July 2010.

16. Abhyudai Singh and João Pedro Hespanha. Lognormal moment closures for biochemical reactions. In Proceedings of the 45th IEEE Conference on Decision and Control, pages 2063-2068, 2006.

17. Murata Tadao. Petri nets: properties, analysis and applications. Proceedings of the IEEE, 77(4):541-580, 1989. 